\title{
Drug resistance bacterial isolates in inpatients at Cocody University Hospital, Abidjan, Côte d'Ivoire in 2014
}

KN Adele

\section{From 3rd International Conference on Prevention and Infection Control (ICPIC 2015)}

Geneva, Switzerland. 16-19 June 2015

\section{Introduction}

Nosocomial infections by the emergence of antibioticresistant bacteria are also a real health problem in limitedresource countries. The high frequency of these infections goes along with increased consumption of antibiotics.

\section{Objectives}

The aim of this study was to determine drug resistance rate of indicator bacteria isolated from nosocomial infections in inpatients.

\section{Methods}

Hospital bass cross-sectional study was conducted on 299 isolates from inpatients of Cocody University hospital from january to december 2014. Bacteriological culture and examination was done following standard microbiological techniques. Drug resistance test was performed by disk diffusion methods against classes of antimicrobials. The data was analysed for descriptive statistics using EPI Info version 6.2 and Microsoft Excel.

\section{Results}

Of the total of 299 isolates, strains were respectively from surgical (30.4\%), pediatrics (18.7\%), medicine (17.7\%), neurology (14\%), pulmonology (11.4\%) and intensive center unit (7\%). Enterobacteriaceae were represented by $60.2 \%$, including Escherichia coli (24.4\%) and Klebsiella pneumoniae (19.1\%). A total of 92 (51.1\%) enterobacteria producing extended spectrum beta-lactamase (ESBL). ESBL have been commonly isolated in pediatric, surgical and intensive care unit, especially in the urine. The rate of resistance to ciprofloxacin were $68.3 \%$ for Enterobacteriaceae. About 62 isolates of Staphylococci (20.7\%), 45 were
Staphylococcus aureus (72.6\%) and $17.8 \%$ were resistant to methicillin (MRSA). Multidrug resistance rate was $4.4 \%$. MRSA were common in surgery especially in suppurations.

Nearly, $10 \%$ of Pseudomonas aeruginosa were resistance to ceftazidime (CRPA). These strains were isolated in intensive care and pediatrics units.

\section{Conclusion}

This study revealed that the rate of drug resistance was high for ESBL. These trends need to be monitored regularly. These data should be taken into account in the strategies against nosocomial infections.

\section{Disclosure of interest}

None declared.

Published: 16 June 2015

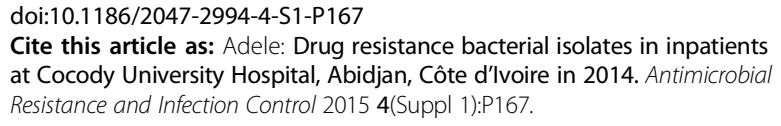

Cite this article as: Adele: Drug resistance bacterial isolates in inpatients at Cocody University Hospital, Abidjan, Côte d'Ivoire in 2014. Antimicrobial Resistance and Infection Control 2015 4(Suppl 1):P167. 